\title{
Understanding Individual Differences for Tailored Smoking Cessation Apps
}

\author{
Jeni Paay, Jesper Kjeldskov, Mikael B. Skov, Lars Lichon and Stephan Rasmussen \\ Department of Computer Science / Research Centre for Socio+Interactive Design \\ Aalborg University, Aalborg East, Denmark \\ \{jeni, jesper,dubois\}@cs.aau.dk
}

\begin{abstract}
Finding ways to help people quit smoking is a high priority in health behavior change research. Recent HCI studies involving technologies using specific quitting techniques such as social support and SMS messaging to help people quit have reported some success. Early studies using computer generated print material report significant success of tailored versus non-tailored material, however, there is limited understanding on what aspects of digitally delivered quitting assistance should be tailored and how. To address this, we have conducted an empirical investigation with smokers to identify perceived importance of different types of help when quitting and the potential role of technology in providing such help. We found that people are highly individual in their approach to quitting and the kind of help they regard as relevant to their situation. Our contribution is a collection of empirically derived themes for tailoring smoking cessation apps to individual quitting needs.
\end{abstract}

\section{Author Keywords}

Smoking cessation; tailoring; individual differences; health behavior change; qualitative research.

\section{ACM Classification Keywords}

H.5.m. Information interfaces and presentation (e.g., HCI):

Miscellaneous.

\section{INTRODUCTION}

Our health is important to us. Over recent years, there has been an increased focus in media, in government policy and in research on preventing the serious risks caused by the effects of behavior choices leading to obesity, smoking, alcoholism, medication abuse and diabetes. In Denmark $25 \%$ of annual deaths are caused by smoking. The habit of smoking can cause serious health issues such as cancer, heart disease, chronic obstructive pulmonary disease, and poor blood circulation. Smoking also has an economical impact on society; the latest numbers showing a yearly cost

\footnotetext{
Permission to make digital or hard copies of all or part of this work for personal or classroom use is granted without fee provided that copies are not made or distributed for profit or commercial advantage and that copies bear this notice and the full citation on the first page. Copyrights for components of this work owned by others than ACM must be honored. Abstracting with credit is permitted. To copy otherwise, or republish, to post on servers or to redistribute to lists, requires prior specific permission and/or a fee.Request permissions from Permissions@acm.org.
}

CHI 2015, April 18 - 23 2015, Seoul, Republic of Korea Copyright 2015 ACM 978-1-4503-3145-6/15/04 ...\$15.00 http://dx.doi.org/10.1145/2702123.2702321 in Denmark of 7.5 billion DKK. Therefore, health organizations are working to reduce the number of smokers; both by preventing people from starting in the first place, and helping those who smoke to reduce and/or quit. By informing the design of more effective smoking cessation apps we aim to support people's transition from smokers to non-smokers, and help them to stay quit.

While hundreds of apps are available for use on various smart phone platforms, HCI research studies on smoking cessation are very few. This means that there is an opportunity to do more research within this domain, of value for both people trying to quit smoking, and developing of smoking cessation apps. The convenience and flexibility of smart phones offer great potentials for creating tailored smoking cessation apps. But in order to provide tailored advice, it is essential to learn about individual habits. Understanding the gains that people express about their smoking habit allows us to design applications that can offer suitable compensations. If personal gains are based on enjoyment, relaxation, pleasure, personal image, rather than nicotine addiction, then what is enough of a substitute for them? A nicotine patch may help some, but might not work for others. From this perspective it makes little sense to aim for "one fits all" solutions.

In this study we specifically look at individual differences in relation to facilitating smoking cessation. To do this we have taken a participatory approach to explore why smokers continue to smoke, in order to remain mindful not only of serious health implications of smoking, but also of the serious challenges that quitting poses to smokers. From this our contribution is a collection of empirically derived themes for tailoring smoking cessation apps to individual quitting needs. Our participants found it very difficult to quit smoking even though they expressed a desire to quit and were aware of the negative health implications if they didn't. We acknowledged their struggles to stay quit and explored with them their ideas on how technology might help them achieve this. Through focus groups and design workshops we discovered 12 themes that should inform the design of smoking cessation apps - making them individualized, monitoring actual smoking behavior, and appropriately compensating and supporting smokers on the personal needs, and the individual cravings, that they each struggle with while trying to quit. 


\section{RELATED WORK}

Smoking addiction and cessation are the focus of an extensive body of research in the health behavior change and psychology fields. Research on addictive behaviors that can inform our understanding of why people smoke, what can motivate them to change this behavior and how to prevent relapse can be found in $[6,19,20,21,22,26,36$, 42, 43]. West and Brown [42] provide a theory of addiction explaining that it is a reflective choice and that the concepts of impulse and self-control play an important role in curbing addictive behavior. Their PRIME theory of motivation to quit is based on a combination of having the capability to do it, the physical and social opportunity to do it, and the motivation to engage in it at that time. This same model is at the centre of Mitchie et al.'s [22] behavior change wheel, which provides a method for characterizing and designing behavior change interventions. It focuses on sources of behavior, interventions functions and policy categories that could enable those interventions. Mitchie and West [20] present the PRIME theory of motivation and the Behaviour Change Wheel as frameworks to government to influence future behavior change interventions and policies. In terms of individual support for smoking cessation, West et al. [43] provide smokers with The SmokeFree Formula to help people to stop smoking. In this book they facilitate smokers taking an individual approach to quitting, acknowledging that there is no one way of quitting that works for everyone. Mitchie at al. [21] acknowledge that little is known about the "active ingredients" of smoking addiction, and amongst other support mechanisms suggest motivation to quit through provision of rewards for abstinence.

The use of technology to support behavior change is increasingly of interest to researchers in both $\mathrm{CHI}$ and behavioral science, for health and sustainability, but the work is "siloed" within the two communities. To help bridge this theoretical gap, Heckler et al. [17] provide guidance for interpreting, using and contributing to behavioral theories. They highlight that much more work is needed in this area before HCI researchers can both utilize and contribute to these theories. In terms of creating working prototypes, Klasnja et al. [18] propose that in evaluating them we need to focus on efficacy evaluations of specific interventions and studies that gain a deeper understanding of people's experiences with the technology.

In looking at specific HCI research on smoking cessation we note that previous studies have indicated a need to investigate the gap between research and practice, as many available smoking cessation apps have not been evaluated for efficacy $[1,2,7]$. In fact, we could not find studies that involved actual smokers in the design process, to better understand their needs within this context. In our review of $92 \mathrm{HCI}$ and health behavior change papers we found that there were very few studies designing technology to support smoking cessation, i.e. [4, 16, 25, 28, 29, 30, 34, 35, 41]. Within these, we did not find any designs created through participatory practices, such as focus groups and design workshops. This inspired us to see if we could find new insights to inform design of smoking cessation technology by involving users in the understanding and envisionment phases of interaction design. In this way, real smokers and their goals and challenges would be behind design considerations and features of the smoking cessation applications, making them more directly relevant to the people who are actually struggling to quit smoking.

In a recent study, we designed and evaluated a smoking cessation application, Quitty. This app offered three different types of content (tips, stories and motivators), which were recommended by either an expert or by fellow smokers in their community [25]. Our findings showed that in order to make an application useful, it should consider users' needs by tailoring content to their situation. This includes consideration of both their quitting stage and demographics. We found that using mobile technology was important so that people could access the app at the times and places that they needed support to stop smoking. Daily SMS messages about new content delivered to the system proved to be both useful and a positive reminder of what they were trying to achieve. Participants were also interested in contributing to and improving the application, based on their own experiences with the app and their own struggle to quit smoking. However, we discovered widely differing wishes in respect to including additional services, such as social media, tracking and games.

In 2009, Abroms et al. [1] reviewed available smoking cessation apps on the iTunes store (47 apps in total) and found little correlation between established guidelines within smoking cessation and the apps available. They recommended that current apps be revised and future apps developed based on evidence-based practices. In 2012, a follow up study by Abroms et al. [2] on popular smart phone apps for smoking cessation (400 apps in total) concluded that these could be improved by better integration with the Clinical Practice Guidelines and other evidence-based practices. Other HCI research studies have created apps with a variety of focuses, in order to support people in quitting smoking, but mostly as targeted materials. Ploderer et al. [28, 29, 30] focused their research on understanding social support amongst peers to inform the design of a "Distract Me" app for mobile phones. Valdivieso-López et al. [41] focused on providing "healthy games" in a mobile app for teenagers. Murnane and Counts [24] analyzed posts on Twitter to discover how people succeed in quitting using social media, and Zhang and Yang [44] studied Facebook posts to discover patterns of behavior that aligned with smokers' quitting stages. A review by Strecher [39] of printed quit smoking materials found that in the majority of the cases, tailored quit smoking material had a statistically significant impact compared to non-tailored materials. While not specifically about smoking cessation, the idea of people crafting their own personal health technologies by Ananthanarayan et al. 
[5] aligns well with our approach of responding to individual differences in health technology design.

Backinger et al. [7] claim that emerging technologies, such as smart phones, have the possibility to offer resourceful means to help within a smoking cessation context. Our interest in the smart phone platform is not only the flexibility and functionality that they provide to make advanced tailoring of content possible, but also the fact that they are likely to be with the smoker at the time and place when they are tempted to have a cigarette, and therefore can provide timely intervention.

\section{EMPIRICAL STUDY}

We conducted an empirical study of smokers' and exsmokers' attitudes towards quitting, and their ideas about how interactive technology might be used to help them quit. The study involved focus groups and design workshops to explore effective tailoring of smoking cessation in a participatory way.

\section{Recruitment and Participants}

We recruited participants over a two-month period through a dedicated web site, which was advertised through social media (Facebook, Twitter and forums), Google AdWords, physical posters and business cards. The website described the project and provided a link to join the study. The national Health and Medicines Authority also helped us recruit by placing an advertisement on their smoking cessation website, and the local Municipality helped recruit participants through their weekly smoking cessation courses. Participants had to be current or previous smokers and own a smart phone (to be able to reflect on the use of this technology in respect to trying to quit).

When agreeing to join the study, each participant completed an online survey with questions about basic demographics (age, occupation, city of residence, etc.) and their smoking habits including stage of quitting based on the TransTheoretical Model of health behavior change (TTM) [32]. This information was used to determine their nicotine dependence using a shortened version of the Fagerströms test [40]. We also collected data on their reasons for smoking, when they usually smoke, if they had ever tried to quit, and their motivations to quit. Lastly, we asked the participants what method (if anything) they had previously used to try and become smoke-free.

We recruited a total of 18 participants from North Jutland, Denmark: 11 males and 7 females. Their ages ranged from 20 to 61 , with an average age of 31 . They had been smoking for between 1 and 40 years, the average for the group being 11.7 years. Out of our participants, 13 had previously tried to quit. In terms of quitting stages, 5 were considering quitting, 5 trying to remain quit, 4 considered themselves as being quit, and 4 were not interested in quitting. For the smokers, their nicotine dependency, based on the Fagerströms test, was: 2 had a score of 0 (no dependence), 6 had a score of 1-2 (very low dependence) and 6 had a score of 3 (low to moderate dependence).

\section{Procedure}

The study was conducted during the spring of 2014, and included 4 focus groups and 2 participant design workshops. The 6 sessions lasted approximately 1.5 hours each, and involved different activities and discussion points.

We chose focus groups to reveal determinants and experiences related to their social smoking behaviors. We were building on the work of Ploderer et al. [28] where interviews were used to gain understanding. We encouraged open discussion on what participants liked about having a cigarette and why they began smoking in the first instance. We then focused on participants' smoking habits including: reasons for smoking, situations and places where they do and do not want to smoke, where they keep their cigarette packets, and what they consider the positives and negatives of smoking. In questions of a personal nature we allowed participants time to write down their response first and then share them with the group in an open forum if they wished to. We were also interested in learning if they had tried to quit before, what they learned from the attempt, what their reasons were for trying, their strategies, the difficulties they faced, and where they would get help, if at all. We also investigated their use of smart phones, choice of general apps and whether they had tried using a smoking cessation app before. We were interested in knowing if social networks could be used in the context of quitting and if $/$ how participants used social media to help them stop smoking. Finally, we showed an example commercial app, "QuitNow - My Quitbuddy" (http://www.quitnow.gov.au) to get their impression of it. This app was chosen because it uses a variety of techniques to help people to stay quit. At the end of the session, participants were presented with two quit smoking messages: a gain-framed one and a loss-framed one. We did this to get their immediate impression of these messages, and see how they responded to positive statements about the benefits of quitting versus negative facts about smoking.

The design workshops involved 9 of the same participants, a mixture of smokers and smokers who had quit. The goal was to gain a better understanding of how the findings from the focus groups could inform the design of smoking cessation apps. We did this by first presenting the findings from the focus group, with examples and explanations to participants. We asked participants to validate the themes as representing important issues of quitting and to add to them if necessary. We then encouraged them to use the final themes as inspirations for sketching designs of possible smoking cessation technologies. Participants were encouraged to discuss and collaborate in creating ideas and sketches and to use technology in their solutions. We asked them to share their ideas with the group explain what inspired their particular design idea. The group then worked together to refine their most promising designs. 


\section{Data Analysis}

For the purpose of analysis, we recorded each session on audio and/or video to be reviewed and transcribed. In the analysis we used open coding [38] to identify distinct concepts and categories. We then iteratively recoded these, looking for relationships amongst the codes. Each session was analyzed separately by two researchers then merged, to achieve concordance, to get different perspectives and ensure that all concepts of importance were included. We found 40 different themes from our merged coding effort. These were further synthesized using affinity diagramming to establish 12 overarching themes. These themes emerged from issues raised about what was perceived as important when quitting smoking and the possible role of technology.

\section{FINDINGS}

Our findings are a collection of empirically derived themes for informing the tailoring of smoking cessation apps to individual quitting needs. In this section we describe these themes, relate them to previous work where relevant, and include design implications where appropriate. We then present ideas from our design workshops as exemplars on how the themes might be used in design. In our findings, we bring new insights on acknowledging and accommodating individual differences in the design of tailored smoking cessation technologies.

\section{Focus Group Findings}

The themes of importance when quitting smoking found in our study are: 1) quitting stage, 2) immediate impact, 3) smoking habits, 4) personalization, 5) flexibility, 6) reminders, 7) self-monitoring, 8) novelty, 9) meaningful rewards, 10) social support, 11) solo struggle, and 12) competition. While some of these are well known (e.g. 1, 3, $4,9,12)$, others have not yet been addressed in the context of designing for smoking cessation (e.g. 2, 5, 8, 11).

\section{1 - Quitting Stage}

Quitting stage describes whether you are thinking of quitting, trying to stay quit, or still want to smoke. Our participants were at different quitting stages, in respect to the six-stage model TTM [32], with 4 of the 6 stages represented. We found a correlation between quitting stage, finding the motivation to quit smoking and the kind of support needed during this stage. Many participants expressed a mismatch between generic quitting information or health risk information and their own quitting needs or stage, and so they ignored it.

This confirms findings from other studies using TTM. Aveyard et al. [6] proved that TTM-based interventions were more successful than interventions not considering the different quitting stages of smokers. The TTM model has been used in several research studies to increase success rates $[3,14,15,25,35,39]$. To achieve the highest efficacy in a smoking cessation app it is important to provide interventions that are tailored to a smokers' quitting stage.

\section{2 - Immediate Impact}

When discussing motivation to quit smoking, and to stay quit, we found that participants faced with an "immediate impact" said they were more likely to accept the advice given. An immediate impact message is one that presents the person with an implication of their current behavior that affects them now. This could be related to health or money, both seen as the main motivators. For example, student participants saw their personal economic state as directly impacting their smoking habits. When they smoked they could see they had less money to spend on other things, having an immediate effect on their lifestyle. One participant said: "the economical aspect is something you can feel right now". Older participants with full-time work were not so concerned by this, as they usually had enough money to comfortably cover their habit. The important immediate impact in their case was generally health-related. They were aware that many years of smoking, coupled with advancing age, placed them at a higher risk of smokingrelated problems.

This finding confirms Morris's [23] guideline: Show people what they could lose. Showing people what they will lose in the short and long-term if they don't change behavior motivates them to change. What constitutes an "immediate impact" differs from person to person, so it is important that tailoring takes this aspect into account.

\section{3 - Smoking Habits}

Smoking can be habit driven. Choosing to have a cigarette can be the result of multiple external factors. Participants had very different smoking habits: some smoked out of enjoyment, others smoked when stressed, some smoked as soon as they walked outside. Participants discussed which cigarette during the day was the most important and whether or not they should smoke in front of children. We found that people's smoking habits and attitudes to smoking were very individual. For example, by knowing a person's smoking pattern, a system could intervene with relevant information/advice/distraction at the time when it is most needed. Being able to judge an individual's critical smoking moment - by time, place or situation - makes it possible to intervene "just-in-time".

West and Brown [42] support the impact of habit on smoking cessation, saying that relapse can be caused by habits that are both unthinking, e.g. lighting up without thinking, and learned behaviors, e.g. rewarding ourselves. Chung et al. mapped the smoking landscape of South Korea in terms of demographics and smoking behavior [9]. They concluded that in knowing peoples smoking habits, appropriate anti-smoking measures could be set, greatly reducing cigarette consumption. Scholl et al. [34] gathered personal smoking habits using sensing technology to prompt smokers to reflect on their own smoking habits with the goal to improve their health. We suggest the same sensing technology could be used to determine people's 
individual smoking habits and provide smoking cessation advice to individuals at the most effective times.

\section{4 - Personalization}

Smoking advice needs to be personalized - the smoker needs to relate to the content as if it was created directly for and about them. Many participants did not feel motivated to quit as they regarded the many health issues associated with smoking as never becoming a problem for them - they felt that it happens to somebody else. One participant said she did not feel motivated when she saw pictures of tar-filled lungs: “... It just doesn't have any effect when those black lungs come up on the screen, because we all think 'Well that isn't my lungs, so how can you say that?" This indicates a need for content to be personalized and as directly relevant to the user and their situation as possible.

Information can be personalized on many different aspects of the smoker's life, such as quitting stage, age, family situation, socio-economic situation, etc. Strecher [39] found that tailored materials seemed to have the greatest impact on pre-contemplators (defined as people thinking about quitting). West et al. [43] provide a printed personalizable quitting program to help people stop smoking. Using an app on an person's smart phone makes it possible to know about their individual needs and to deliver content that matches their situation and quitting stage, just as face-to-face counseling can [39].

\section{5 - Flexibility}

Smokers have very different needs and approaches when it comes to quitting. They need different tools, information and compensations at different times in the process and at different times in the day to deal with the difficult and important activity of giving up cigarettes. All of our 18 participants had different approaches to quitting, no matter what quitting stage they were at. Some preferred going "cold turkey", while others wanted to reduce their cigarette intake gradually. When participants were shown the QuitBuddy app during the focus session, and it's built in "distraction" game, one said, "... I would need to be able to say 'I want WordFeud instead' and then just easily via a few steps be able to have WordFeud there." So a distraction needs to interesting to the individual and easy to access if it is going to distract them from smoking. Any smoking cessation app hoping to facilitate peoples' quitting approaches needs to be flexible enough to adapt to the user's current needs.

\section{6 - Reminders}

SMS messages have been successfully used to remind people to use their smoking cessation app. However, we found that reminders also bring the idea of smoking cigarettes to the front of people's thoughts - and in the worst case, actually prompt them to smoke. As one participant said, "It must not remind people of having a smoke. Enough is doing that already. If I had an app that kept giving me messages, I would just think 'I could really use a cigarette right now',". In discussing phone usage with participants it became apparent that engaging with their mobile phones was often dependent on their context and happened mostly when they were out. Reminders to use a smoking app needs to be sent out when and where a smoker tends to crave cigarettes to avoid making them think about smoking when they otherwise wouldn't, and to help them resist.

Fogg and Allen [12] found the use of texting to persuade health behavior change effective, using SMS messages to remind and notify people in regard to specific health topics. Roubroeks et al. [32] studied the forms that such notifications should take in order to achieve maximum efficacy. Both studies confirm the effectiveness of reminders. In our recent study [25] we found that some participants responded positively to messages that were sent at different times of the day, surprising them and making it difficult for them to plan to avoid the message. It was important that these messages were sent by a person, rather than computer generated.

\section{7 - Self-Monitoring}

Self-Monitoring is a well-known technique in persuading people to change their health behavior [11]. By selfmonitoring we mean both data input by the user and data about that user measured by the system. When shown the QuitBuddy app the participants all liked the idea of the selfmonitoring elements. Based on daily estimates input by users, QuitBuddy provides a smoke-free timer, the total amount of money the user has saved, and the amount of tar avoided. One participant said: "I think that the timer is good, but it is because I see the timer like somewhat a high score and as soon as you are having a cigarette, the number hits zero and you have to start all-over again." "Seeing" a representation of how much they smoked had a motivating effect toward not smoking. The money saved by participants was an "eye-opener" for them, which they also said would be a strong motivator to reduce their smoking.

"Quantified Selfers" [8] have shown that being aware of various aspects of their self, by counting things such as steps taken during a day using devices such as pedometers, helps them reflect on their own health and well being. Selfmonitoring could therefore be used to help people understand their own smoking habits and create strategies to cope with cravings [36]. Self-monitoring was used by Ali et al. [4] to automatically detect when people were smoking using a device measuring respiration. Scholl et al. [34] created a lighter that registers when the user is lighting a cigarette to count the number of cigarettes smoked in a day. These technologies for measuring different aspects of a person's activity could be used to feed individual data into a smoking cessation app, which would then be tailored to respond appropriately. The forms that this response could take, such as visualizations of smoking behaviors, calculated compensations in the form of financial or health gains, or stories and tips tailored to a persons behaviors, are all design possibilities and based on actual behaviors. 


\section{8 - Novelty}

Presenting people with new and surprising information attracts attention and sparks interest. Our participants said it would be better if smoking cessation information was delivered in more creative ways in terms of content, technology and interaction. All agreed that information that is already known to them is easily ignored. For example, smoking cessation campaigns often target negative health impacts of smoking, but this is information that smokers are well aware of and tired of hearing about, "It is fine that we have some smoking cessation campaigns, but it is not something you should apply in the application that smokers should use, because they already know it." Smokers feel bombarded and underwhelmed by general health warnings and scare tactics. They said that knowing these impacts has not yet stopped them from smoking - they need something else, something they don't already know.

Designers and developers of smoking cessation apps need to provide novelty in the way that they distract, compensate and motivate smokers. We discovered in our study that the needs of smokers quitting are both more complex and subtler than those represented in the literature reviewed, and that this is strongly influenced by the different needs of individuals in terms of what they already know and what has an impact on them personally.

\section{9 - Meaningful Rewards}

People quitting smoking need suitable compensations to successfully counteract the personal gains that they get from smoking. These "rewards" for not smoking are highly individual. It needs to be something that holds value and meaning for that person. Participants saw meaningful rewards as a huge motivator, both in terms of reducing current intake of cigarettes, and staying quit. Examples of suitable rewards discussed included money "earned" from not smoking, items that could be bought with that money, specific health improvements, and personal achievements. One participant said, "It was just so nice to be able to go to the bike store and say 'I am going to get a proper bike' which is what I have now." Another participant stressed that it is important that the reward is greater than the satisfaction gained from smoking, "My reward should be bigger than how delicious I think it is to smoke." Rewards need to be defined by the smoker, so that they are meaningful and significant enough for that person to compensate for smoking a cigarette.

Mitchie et al. [21] propose reward as an important part of individual behavior support for smoking cessation. Other studies have shown that financial rewards can be highly motivational. A study conducted with pregnant women who were given monetary incentives to stay off cigarettes showed that one-third had completely abstained from smoking by the twelfth week of the study [37]. Snuggs et al. [36] discovered that stimulation obtained from rewards increased within a week of stopping smoking and after 4 weeks had increased even more.
10 - Social Support

Social support for smoking cessation using online forums and social networking sites presents an interesting alternative to stand-alone apps in terms people getting support and stories from others in a similar situation. Participants' views on the usefulness of social support while quitting differed widely. Most said they would not use Facebook for social support, as they regarded the content on social networking sites as generally banal, while quitting smoking was perceived as a very important and personal quest, which they did not want to share openly. They generally felt that adding a social aspect would make them become dishonest about their struggle to avoid the embarrassment of failing. They also felt that telling their friends and family about their quitting attempt added unnecessary pressure and became a stressing factor, which in a worst-case scenario would make them start smoking again. Participants agreed that social support works best when shared with strangers.

Studies of different online smoking cessation groups give understanding of the patterns of posting and responses between people trying to quit [24, 29, 42, 44]. Ploderer et al. [27] found that some individuals who participate in Facebook groups take on leadership roles to support others, depending on their own quitting stage. Our study found that any social support in the app should allow for anonymity.

\section{1 - Solo Struggle}

Quitting smoking is a very personal challenge, and our participants felt that at some point in time you just have to "do it alone". Rather than adding a social aspect to the struggle, some felt they would much rather take on the challenge by themselves, as one person clarified, "because others cannot fight for you". Another participant explained, "There is nothing they can say that influences me. It is only my own expectations that influence me". In fact, making it a personal struggle would motivate smokers to stay abstinent, "Okay now I have been smoke-free for 4 days by myself",". Being able to do it alone becomes a reward in itself.

It was very important to our participants that any app gave them the flexibility to tailor their own quitting attempt in respect to doing it by themselves, and what they needed to achieve this.

\section{2 - Competition}

Competition was a subject of discussion in all focus groups. Participants felt that it was highly motivational to be involved in competition, either against others or against themselves. One participant explained, "But when you think that you want to have a cigarette and when you think 'no I will take a look at my score, making sure that I do not break it'. It is like a game, where you always gain new levels, and when you take a cigarette you lose levels. Then it is like 'Ah crap'... I want to win this competition against others and for how long can I keep off the smokes compared to others?" indicating that competition can give smokers an incentive to stay quit. 
In a study by Cummings et al. [10] smokers were placed in a worksite Stop-Smoking Contest in which cash prizes were awarded for quitting smoking. The study showed that onethird of participants managed to stay abstinent after 3 months, indicating that competition and/or monetary gain are strong motivators to stop smoking.

\section{Design Workshop Sketches}

In our two design workshops we presented the 12 quit smoking themes to 9 participants from the focus groups. We did this to provide inspiration for generating technology design ideas to support quitting. The outcomes from these workshops were 15 different sketched designs. These designs were highly diverse, addressing different opportunities, challenges and requirements for people at different quitting stages. Most involved the use of mobile phones, either as the main tool or in collaboration with other technologies. This was due to the easy accessibility of their smart phones as a physical prop at the design session. As an illustration of the diversity of ideas, and to demonstrate how our quit smoking themes can inform design ideas we briefly describe three design ideas from the workshops: Show The Losses and Gains for Me; Automated Self-Monitoring and Personalized Interactive Cigarette Package.

\section{Show Losses and Gains for Me}

The Show Losses and Gains for Me design idea displays timelines showing the health and economic consequences of the user's smoking choices. The system is primed by entering personal interests, goals, cigarette brand and number of cigarettes smoked per day in order to give individualized feedback. The number of cigarettes is used to calculate their current position on the timeline in respect to projected losses or gains from increasing, maintaining or reducing their cigarette intake. If they reduce their smoking, they move along a "Rewards" timeline toward a bigger reward (e.g. a holiday). If they continue smoking, they move along a "Health Consequences" timeline towards declining health (e.g. cancer). This makes the user aware of things to be lost or gained depending on their smoking habits. As one participant said, "I want the system to say that you could have had all this now, making me think about my habits."

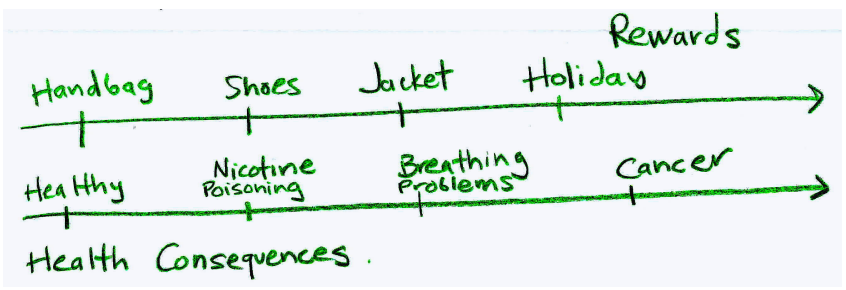

Figure 1. Sketch showing examples of losses and gains from smoking on a personal timeline

In this sketch there are two timelines. There is an economic rewards timeline showing personally chosen compensations for not smoking. The other timeline shows health consequences important to this person. Included in the design is the idea that the rewards could be shown as images of the actual items. By having data and representations based on personal habits, health concerns, and chosen rewards, it becomes more pertinent to the user and provides stronger motivation to not smoke that next cigarette.

\section{Automated Self-Monitoring}

In the Automated Self-Monitoring design, the user wears a wristband measuring their pulse rate as an indicator of when they are smoking. Smoking causes an acute elevation of blood pressure [31], although alcohol intake, body mass, age and gender can all affect the blood pressure-smoking relationship. It is also difficult to measure a single cigarette against several smoked in one session. So, given that it is a complex interrelationship, this design is based on the ability to accurately register individual cigarettes using temporary blood pressure elevation. This was done to avoid the unreliability of people retrospectively remembering the number of cigarettes smoked during the day. The wristband is wirelessly connected to the user's smart phone, to display their data. The user can then compare their current smoking patterns with past patterns, encouraging them to reflect on their actual smoking habits.
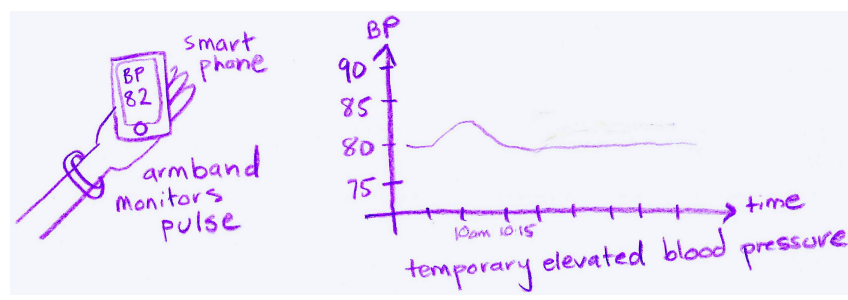

Figure 2. Sketch of the Automated Self-Monitoring Wristband

\section{Personalized Interactive Cigarette Package}

The Personalized Interactive Cigarette Package was very popular with design workshop participants; they found it novel and surprising. The cigarette pack with an interactive screen on the front would showing information about the number of cigarettes left in the pack, illustrating the implications of smoking this number of cigarettes as a health consequence.

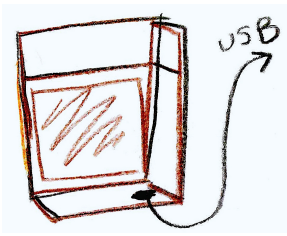

$$
\begin{aligned}
& \text { (1) how do you fill it? } \\
& \text { (2) how does it know how many } \\
& \text { are inside? } \\
& \qquad \text { weight }
\end{aligned}
$$

Figure 3. Sketch of the Interactive Cigarette Pack.

This idea was partly inspired by the kind of anti-smoking information that can already be seen on many cigarette packages showing the health risks of smoking. Design workshop participants stressed the importance of being able to personalize the messages and images on the screen to make them more motivational. 


\section{DISCUSSION}

Tailored smoking cessation apps present a unique challenge for design. While there are successful health behavior change apps that encourage people to $d o$ something healthy (e.g. exercise), a different approach is needed when wanting to encourage people not to do something.

It is important to understand that quitting smoking is not as straightforward as just stopping smoking. Many people "stop smoking" several times during their attempt to quit, but find themselves starting again. In these cases the quitting process then starts all over again. During a quitting attempt, smokers can transition and iterate through the different quitting stages at different rates, in order to become smoke free on a long-term basis. We need to acknowledge that for some, just reducing the number of daily cigarettes in a maintainable way is a victory - and a step in the right direction. Reducing the number of cigarettes per day is also important in achieving improved health and reducing the risks of cancer [15, 39].

\section{Designing for Individual Differences}

By presenting insight into the character and importance of individual differences in smoking cessation, we aim to influence the design of future smoking cessation apps. As an example of this, we have used our identified themes of smoking cessation to envision smoking cessation apps that are tailored to individuals and their personal quitting needs, and hence more useful during all stages of quitting.

Related studies have shown that tailoring information when helping people quit smoking is important $[3,5,14,15,25$, $34,35,39]$. In our study we found that tailoring is a complex and fine-grained concept with multiple facets to be considered in concert including the twelve themes presented: a persons quitting stage; implications that have the most immediate impact on a person; their individual smoking habits, including time, place and triggers; personalized advice they can relate to; flexible choice of quitting tools; reminders that come at the most appropriate time for a person; self-monitoring data about their own behavior; advice or facts that are new to a person; rewards and compensations that hold meaning for a person; and the option of social support or doing it solo at different times. While these themes can be related to the work and findings of others, and some of them are already known, our contribution is in bringing them forward as a collection of insights on the challenges of quitting smoking and the design of technology to support this. Together, they outline a design space for considering individual changes in tailored smoking cessation apps.

\section{Monitoring Actual Smoking Behavior}

From the presented themes we found that, in particular, self-monitoring using technology is an interesting approach, and one that is being used in different behavior change research projects, e.g. [4, 8, 34, 36]. During focus group and design workshop discussions, smokers expressed the necessity of having an automated system to monitor their smoking behavior. They claimed that it was difficult to manually enter data, given that smoking often happens in social situations such as parties, where it would not only be awkward to enter that data, but embarrassing. Relying on the memory of such events and entering the data retrospectively was perceived to be a completely unreliable method of counting cigarettes smoked. They felt this made the monitoring and reflecting on habits exercise invalid, as it would not represent true data about their behavior.

An interesting challenge that came out of our study, is that the very use of a quit smoking app tends to bring smoking to the front of a smokers mind, making them think about cigarettes and even increasing their desire to have one. Apps that run on a their smart phone, sending reminding SMS messages to smokers to use the app or reflect on their smoking habits run the risk of actually prompting the very cravings that they are trying to avoid. This raises an interesting issue, when designing technology for this context: How can we create a technology to help people quit, without reminding them about cigarettes?

\section{Providing Appropriate Compensation}

Another issue is related to the idea of rewards for not smoking that are significant enough to compensate for the cigarette they are replacing. If people are smoking for pleasure, we found that they had no trouble listing things and experiences that they would find a suitable substitute for a cigarette, ranging from a new hat to making love with their partner. However, if people are using smoking as a form of self-medication against stress or social awkwardness, a suitable compensation is much more difficult to define. Even more difficult is finding a role for technology to play in this situation. Turning to your smart phone may simply waste time that you are already feeling pressured about, and it certainly does not help the social confidence of a person already feeling awkward.

\section{Methodological Concerns}

In reflecting on our methodological approach, we found that the topic of people's smoking habits was very sensitive, making it difficult to investigate and gain the trust of our participants. One participant approached us at the end of a focus group session and told us that answering one of the questions during the session was very painful for her. In retrospect, it would have been useful to add an empirical method that creates more individual space for participants sharing sensitive or taboo topics or to capture data from them while in their context of smoking, such as cultural probes [13], giving participants the opportunity to express their smoking cessation issues in a more private way.

\section{Limitations}

We acknowledge that our findings are based on a qualitative study with fewer participants than would be involved in a quantitative clinical study. As the study aims to understand the phenomenon of individual differences in smoking cessation, rather than test the effectiveness of a particular treatment or app, we have deliberately chosen a 
qualitative approach. As per the qualitative research method paradigm, this has allowed us work very closely with our study participants, and to gain richer insights into their thoughts and motivations in relation to quitting smoking.

\section{CONCLUSIONS}

We have conducted an empirical investigation with smokers to identify and understand what types of assistance are perceived and experienced as important when trying to quit, and explore the role that technology can potentially play in providing such assistance. To do this we have taken a participatory approach of working closely with a group of smokers and ex-smokers through focus groups and design workshops. Our study showed that people are highly individual in their approach to quitting and in the kind of help they regard as relevant to their situation. To design effective smoking cessation applications, these individual differences should be at the core of our designs. In tailoring smoking cessation apps it is important to consider: 1) quitting stage, 2) immediate impact, 3) smoking habits, 4) personalization, 5) flexibility, 6) reminders, 7) selfmonitoring, 8) novelty, 9) meaningful rewards, 10) social support, 11) solo struggle, and 12) competition. Our contribution is in bringing these themes forward as a collection is that jointly outline a design space for considering individual differences in tailored smoking cessation apps. As an illustration of the use of the identified themes in technology design, we have presented and discussed three different design ideas for tailored smoking cessation apps produced by our participants.

Our findings present new insights, and confirm findings from previous related research on supporting health behavior change. We have put previous findings into the context of tailored smoking cessation apps, have contributed with a deeper understanding of important factors when trying to quit smoking and elaborated on how to cater for individual differences when providing tailored quitting assistance.

\section{ACKNOWLEDGMENTS}

We thank all study participants for taking time to provide insights into the life of being a smoker and trying to quit. We also thank the Danish National Health and Medicines Authority and the Municipality of Aalborg for their help in recruiting participants for the study.

\section{REFERENCES}

1. Abroms, L., Padmanabhan, N., Thaweethai, L. and Phillips, T. iPhone Apps for Smoking Cessation: A Content Analysis. Am. J. of Preventive Medicine, 40, 3 (2011), 279-285.

2. Abroms, L., Westmaas, L., Bontemps-Jones, J., Ramani, R. and Mellerson, J. A Content Analysis of Popular Smartphone Apps for Smoking Cessation. Am. J. of Preventive Medicine, 45, 6 (2013), 732-736.
3. Ahijevych, K. and Wewers, M. Processes of Change Across Five Stages of Smoking Cessation. Addictive Behaviors, 17 (1992), 17-25.

4. Ali, A., Hossain, S., Hovsepian, K., Rahman, M., Plarre, K. and Kumar, S. mPuff: Automated Detection of Cigarette Smoking Puffs from Respiration Measurements. In Proc. IPSN '12, ACM Press (2012), 269-280.

5. Ananthanarayan, S., Lapinski, N., Siek, K. and Eisenberg, M. Towards the Crafting of Personal Health Technologies. In Proc. DIS '14, ACM Press (2014), 587-596.

6. Aveyard, P., Massey, L., Parsons, A., Manaseki, S. and Griffin, C. The effect of Transtheoretical Model based interventions on smoking cessation. Social Science and Medicine, 68 (2009), 397-403.

7. Backinger, C. and Auguston, E. Where There's an App There's a Way? Am. J. of Preventive Medicine, 40, 3 (2011), 390-391.

8. Choe, E., Lee, N., Lee, B., Pratt, W. and Kientz, J. Understanding Quantified-Selfers' Practice in Collecting and Exploring Personal Data. In Proc. CHI '14, ACM Press (2014), 1143-1152.

9. Chung, W., Kim, H., Lim, S., Lee, S. and Cho, K. Factors influencing cigarette smoking and quantified implications for anti-smoking policy: evidence from South Korea. Int. Journal of Public Health, 54, 6 (2009), 409-419.

10. Cummings, K., Hellmann, R. and Emon, S. Correlates of Participation in a Worksite Stop-Smoking Contest. Journal of Behavioral Medicine, 11, 3 (1988), 267-277.

11. Fogg, B. Persuasive Technology. Morgan Kaufmann, 2003.

12.Fogg, B. and Allen, E. 10 Uses of Texting to Improve Health. In Proc. Persuasive '09, ACM Press (2009), Article 38.

13. Gaver, B., Dunne, T. and Pacenti, E. Design: Cultural probes. Interactions, 6, 1 (1999).

14. Girma, E., Assefa, T. and Deribew, A. Cigarette smokers' intention to quit smoking in Dire Dawa town Ethiopia: an assessment using the Transtheoretical Model. BMC Public Health, 10 (2010).

15. Godtfredsen, N. and Prescott, E. Effect of Smoking Reduction on Lung Cancer Risk. J. of the Am. Medical Association, 294, 12 (2005), 1505-1510

16. Graham, C., Benda, P., Howard, S., Balmford, J., Bishop, N. and Borland, R. "heh - keeps me off the smokes...": Probing Technology Support for Personal Change. In Proc. OzCHI'06, ACM Press (2006), 221228.

17. Heckler, E., Klasnja, P., Froehlich, J. and Buman, M. Mind the theoretical gap: interpreting, using, and 
developing behavioral theory in HCI research. In Proc. CHI'13, ACM Press (2013), 3307-3316.

18. Klasnja, P., Consolvo, S. and Pratt, W. How to evaluate technologies for health behavior change in $\mathrm{HCI}$ research. In Proc. CHI'11, ACM Press (2011), 30633072 .

19. Marlatt, G. and Donovan, D. Relapse Prevention. Guilford Press, 2005.

20. Michie, S. and West, R. Behaviour change theory and evidence: a presentation to Government. Health Psych Review, 7, 1 (2013), 1-22.

21. Michie, S. Hyder, N., Walia, A. and West, R. Development of a taxonomy of behaviour change techniques used in individual behavioural support for smoking cessation. Addictive Behaviors, 36 (2011), 315319.

22. Michie, S., van Stralen, M. and West, R. The behaviour change wheel: A new method for characterising and designing behaviour change interventions. Implementation Science, 6 (2011), 42.

23. Morris, M. Motivating Change with Mobile: Seven Guidelines. Interactions, 19, 3 (2012), 26-31.

24. Murnane, E. and Counts, S. Unraveling Abstinence and Relapse: Smoking Cessation Reflected in Social Media. In Proc. CH I'14, ACM Press (2014), 1345-1354.

25. Paay, J., Kjeldskov, J., Brinthaparan, U., Lichon, L., Rasmussen, S., Srikandaraja, N., Smith, W., Wadley, G., Ploderer, B. Quitty: Using Technology to Persuade Smokers to Quit. In Proc. NordiCHI'14, ACM Press (2014).

26. Park, E., DePue, J., Goldstein, M., Niaura, R., Harlow, L., Willey, C., Rakowski, W. and Prokhorov, A. Assessing the Transtheoretical Model of Change Constructs for Physicians Counseling Smokers. Annals of Behavioral Medicine, 25, 2 (2003), 120-126.

27.Ploderer, B., Smith, W., Howard, S. Pearce, J. and Borland, R. Patterns of Support in an Online Community for Smoking Cessation. In Proc. C\&T'13, ACM Press (2013), 26-35.

28. Ploderer, B., Smith, W., Howard, S. Pearce, J. and Borland, R. Things You Don't Want to Know About Yourself: Ambivalence About Tracking and Sharing Personal Information for Behavior Change. In Proc. OzCHI'12, ACM Press (2012), 489-492.

29. Ploderer, B., Smith, W., Howard, S. Pearce, J. and Borland, R. Introducing the Ambivalent Socialiser. In Proc. CHI'12, ACM Press (2012), 1511-1514.

30. Ploderer, B., Smith, W., Pearce, J. and Borland, R. A Mobile App Offering Distractions and Tips to Cope With Cigarette Craving: A Qualitative Study. JMIR mHealth uHealth 2, 2 (2014), e23.
31.Primatesta, P., Falaschetti, E., Gupta, S., Marmot, M. and Poulter, N. Association Between Smoking and Blood Pressure. Hypertension, 37 (2001), 187-193.

32. Prochaska, J. and Velicer, W. The Transtheoretical Model of Health Behavior Change. Am. J. of Health Promotion, 12, 1 (1997), 38-48.

33. Roubroeks, M., Ham, J. and Midden, C. When Artificial Social Agents Try to Persuade People: The Role of Social Agency on the Occurrence of Psychological Reactance. Int. J. of Social Robotics, 3, 2 (2011), 155165.

34. Scholl, P., Kücükyildiz, N. and Laerhoven, K. Demo: When Do You Light a Fire? Capturing Tobacco Use with Situated Wearable Sensors. In Proc. UbiComp'13, ACM Press (2013), 1309-1310.

35. Shimoda, T. and Stapel, L. Increasing Relevance of Smoking Cessation Messages in an Online Software Agent Environment. In Proc. HICSS'06, Vol. 5, IEEE (2006), 89a.

36. Snuggs, S. and Hajek, P. Responsiveness to reward following cessation of smoking. Psychopharmacology, 225, 4 (2013), 869-873.

37. Springer Healthcare News. Financial reward stubs out smoking habit. Published online, Springer Healthcare (2012).

38. Strauss, A. and Corbin, J. Basics of Qualitative Research: Techniques and Procedures for Developing Grounded Theory. Sage Publications, 1998.

39. Strecher, V. Computer-tailored smoking cessation materials: A review and discussion. Patient Education and Counseling, 26, 2 (1999), 107-117.

40. The Fagerströms Test. http://www.health.wa.gov.au/smokefree/docs/Fgerstrom _Test.pdf. Government of Western Australia Department of Health.

41. Valdivieso-López, E., Flores-Mateo, G., Molina-Gómez, J., Rey-Reñones, C., Uriarte, M.B., Duch, J. and Valverde, A. Efficacy of a mobile application for smoking cessation in young people: study protocol for a clustered randomized trial. BMC Public Health 2013, 13 (2013).

42. West, R. and Brown, J. Theory of Addiction. Wiley Blackwell, 2013.

43. West, R. with Smyth, C., West, J. The SmokeFree Formula. Orian, 2013.

44.Zhang M. and Yang, C. The Effectiveness of Smoking Cessation Intervention on Facebook: A Preliminary Study of Posts and Users. In Proc. ICSH'13, Springer (2013), 7-17. 